City University of New York (CUNY) CUNY Academic Works

2015

\title{
A model microfluidics-based system for the human and mouse retina.
}

Shawn Mishra

CUNY City College

Ankush Thakur

CUNY City College

Stephen Redenti

CUNY Lehman College

Maribel Vazquez

CUNY City College

\section{How does access to this work benefit you? Let us know!}

More information about this work at: https://academicworks.cuny.edu/cc_pubs/329

Discover additional works at: https://academicworks.cuny.edu

This work is made publicly available by the City University of New York (CUNY).

Contact: AcademicWorks@cuny.edu 


\title{
A model microfluidics-based system for the human and mouse retina
}

\author{
Shawn Mishra ${ }^{1} \cdot$ Ankush Thakur $^{1} \cdot$ Stephen Redenti $^{2} \cdot$ Maribel Vazquez $^{1}$
}

Published online: 16 October 2015

(C) Springer Science+Business Media New York 2015

\begin{abstract}
The application of microfluidics technologies to the study of retinal function and response holds great promise for development of new and improved treatments for patients with degenerative retinal diseases. Restoration of vision via retinal transplantation therapy has been severely limited by the low numbers of motile cells observed post transplantation. Using modern soft lithographic techniques, we have developed the $\mu$ Retina, a novel and convenient biomimetic microfluidics device capable of examing the migratory behavior of retinal lineage cells within biomimetic geometries of the human and mouse retina. Coupled computer simulations and experimental validations were used to characterize and confirm the formation of chemical concentration gradients within the $\mu$ Retina, while real-time images within the device captured radial and theta cell migration in response to concentration gradients of stromal derived factor (SDF-1), a known chemoattractant. Our data underscore how the $\mu$ Retina can be used to examine the concentrationdependent migration of retinal progenitors in order to enhance current therapies, as well as develop novel migration-targeted treatments.
\end{abstract}

Keywords Retina $\cdot$ Progenitor $\cdot$ SDF-1 $\cdot$ Diffusion · Migration

Maribel Vazquez

vazquez@ccny.cuny.edu

1 Department of Biomedical Engineering, City College of New York, New York, NY, USA

2 Department of Biology, Lehman College, New York, NY, USA

\section{Introduction}

Degenerative retinal diseases, such as age-related macular degeneration (AMD) and retinitis pigmentosa (RP), are often characterized by the slow and progressive loss of photoreceptor cells, which are retinal cells that convert photons into electrical signals (Rivolta et al. 2002; Margalit and Sadda 2003; Kulkarni and Kuppermann 2005). The inability of the retina to repair and regenerate damaged photoreceptor (PR) cells results in permanent loss of vision. Recent studies have demonstrated abilities to repopulate the retina with functional PR cells via transplantation of post-natal or stem cell-derived retinal progenitor cells (RPCs) (Klassen et al. 2004; MacLaren et al. 2006; Meyer et al. 2006; Redenti et al. 2009). Functional outcomes currently indicate that the amount of restored vision heavily depends upon the migration of transplanted cells from insertion in the sub-retinal space to the outer nuclear layer (ONL) of the retina (Tomita et al. 2005; Meyer et al. 2006; $\mathrm{Ng}$ et al. 2007; Tao et al. 2007). A significant hurdle to functional photoreceptor cell replacement is the restricted number of viable cells that migrate into damaged retina during transplantation (Tomita et al. 2005; West et al. 2009; Lakowski et al. 2010). Further, limited optical accessibility and transparency of the retina and pigment epithelium (RPE) make it difficult to observe movement of motile, transplanted cells within the retina in vivo (Nickerson et al. 2013). The use of explanted retinal tissue is limited for the study of chemotaxis due to rapid degeneration of neural tissue (Mohlin and Johansson 2011). Hence, to functionally repopulate the retina, it is vital to characterize and augment the migration of transplantable RPCs. To achieve this goal requires, first, understanding of the chemotactic mechanisms by which the RPCs migrate into the retina and, second, determining to what extent these mechanisms can be targeted to control and direct RPC migration. A microfluidics-based system provides an excellent platform 
with which to examine the molecular signaling guiding migration of transplanted RPCs, as such devices are comprised of tightly-controlled microenvironments that facilitate detailed and quantitative characterization of cellular processes and behaviors (Meyvantsson and Beebe 2008).

A number of microfluidics systems have been developed over the past decade for the study of cells derived from the central nervous system (CNS) (Stieglitz et al. 2000; Morin et al. 2006; Park et al. 2009). These systems have been designed to examine multiple phenomena, including neuronal and glial co-culture (Stieglitz et al. 2000; Morin et al. 2006; Park et al. 2009; Hosmane et al. 2010), axon degeneration (Park et al. 2006; Vahidi et al. 2008; Kim et al. 2012), and drug delivery (Shawgo et al. 2002; Lavan et al. 2003; Dittrich and Manz 2006), among others, while utilizing a milieu of engineering techniques, such as forced flow (Pan et al. 2005; Estermann et al. 2013), micropatterning (Schlosshauer et al. 1999; Camelliti et al. 2006), diffusion-based flow, 2D cultures, and 3D cultures to do so (Meyvantsson and Beebe 2008; Puleo et al. 2009; Park et al. 2012). However, there have been a limited number of systems designed to model the visual system (Schlosshauer et al. 1999; Puleo et al. 2009), and even fewer that further our understanding of cell migration and signaling within the retina (Schlosshauer et al. 1999; Steedman et al. 2010).

In this study, we have developed a convenient, diffusionbased microfluidics system that replicates the geometry and scale of the mouse and human retina, called the microRetina ( $\mu$ Retina). Our system is designed to facilitate examination of cell responses to steady-state concentration profiles and concentration gradients to provide critical insight into RPC migratory response, so as to aid in transplant therapies for retinal diseases. RPCs were cultured in these retinal-mimetic systems for $48 \mathrm{~h}$ to enable study of cell migratory behavior. RPC responses to the exogenous growth factor stromal cell-derived factor 1 (SDF-1), a known chemoattractant for CNS-derived cells, was measured in the presence of different concentration gradients of SDF-1. Our results illustrate that there is a threshold concentration gradient which will elicit directed migratory behavior from RPCs.

\section{Methods}

\subsection{System fabrication}

The $\mu$ Retina design is fabricated using conventional photolithography and elastomeric molding, as shown in Fig. 1. A patterned design was imprinted onto a 4-in-diameter silicon wafer, as described previously by our laboratory (Kong et al. 2011). The negative photoresistive polymer SU-8 2075 (Sigma Aldrich) was first spin-coated at $1000 \mathrm{rpm}$ for $30 \mathrm{~s}$ onto the wafer surface. The wafer was then pre-baked, on a hot plate, at $65^{\circ} \mathrm{C}$ for $5 \mathrm{~min}$, followed by an additional $15 \mathrm{~min}$ at $95{ }^{\circ} \mathrm{C}$. The wafer substrate was next irradiated with UV light (wavelength $\lambda=360 \mathrm{~nm}$ ) with an intensity of between 215$240 \mathrm{~mJ} / \mathrm{cm}^{2}$ for $23 \mathrm{~s}$, as seen in Fig. 1b. Afterwards, the wafer was post-baked on a $65^{\circ} \mathrm{C}$ hot-plate for 5 mins followed by 10 mins on a $95{ }^{\circ} \mathrm{C}$ hot-plate. Lastly, the substrate was immersed in a complementary developer solution (Microchem, Newton, MA), leaving the desired $100-\mu$ m-thick pattern on the wafer surface.

The patterned substrate was then placed into a custommade aluminum housing, where $20 \mathrm{~mL}$ of polydimethylsiloxane (PDMS) was poured atop the wafer to produce an elastomer of 2-3 mm thickness, as seen in Fig. 1c. An aperture of $100 \mu \mathrm{m}$ diameter was then created through each PDMS reservoir to generate two injection ports used for addition of cell media, biomolecules and cells as needed.

To ensure permanent attachment, the PDMS elastomer was treated with oxygen plasma for a total dosage of $30 \mathrm{~s}$ and attached mold-side down to an $\mathrm{O}_{3}$-plasma treated glass microscope slide (VWR 48300-036), to produce the finished microdevice, as seen in Fig. 1d and e.

\subsection{Computational model}

A two-dimensional numerical simulation of molecular transport within the $\mu$ Retina system was performed using a finite element model of the device created using COMSOL Multiphysics 4.3 (COMSOL Inc., Burlington, MA). An effective diffusivity of $2.14 \times 10^{-7} \mathrm{~cm}^{2} / \mathrm{s}$ was used to model transport of SDF-1 within the retina, as detailed previously by our group (Kong et al. 2010; Unachukwu et al. 2013). The effective diffusivity outside of the retinal space was set to $1 \times$ $10^{-6} \mathrm{~cm}^{2} / \mathrm{s}$, to better approximate the effective coefficient of SDF-1 within water. All physical boundaries of the microsystem were regarded as insulated boundaries of mass transfer and momentum transport as only trace amounts of Dextran were observed to permeate through PDMS surfaces over $24 \mathrm{~h}$. Mesh quality was adjusted to 4800 mesh points for the mouse model and 7000 mesh points for the human model, to ensure simulation accuracy.

\subsection{Gradient formation}

An inverted epifluorescent microscope (Nikon) with a $10 \times$ objective (Nikon, Morrell Instrument Co. Inc, Melville, NY) was used to image the $\mu$ Retina systems via CCD camera (Spot Insight, Diagnostic Instruments, Inc) utilizing SPOT Software (Spot 5.0). A $2 \%$ hyaluronan (HA) solution was injected into the $\mu$ Retina to create a $3 \mathrm{D}$ matrix that mimics the interphotoreceptor matrix (IPM) within the retina (Pitkänen et al. 2005; Hussain et al. 2010). Both reservoirs were then filled with media. The Source reservoir contained $25 \mathrm{uL}$ of $100 \mathrm{ng} / \mathrm{mL}$ of $10 \mathrm{kDa}$ dextran conjugated to alexafluora red 


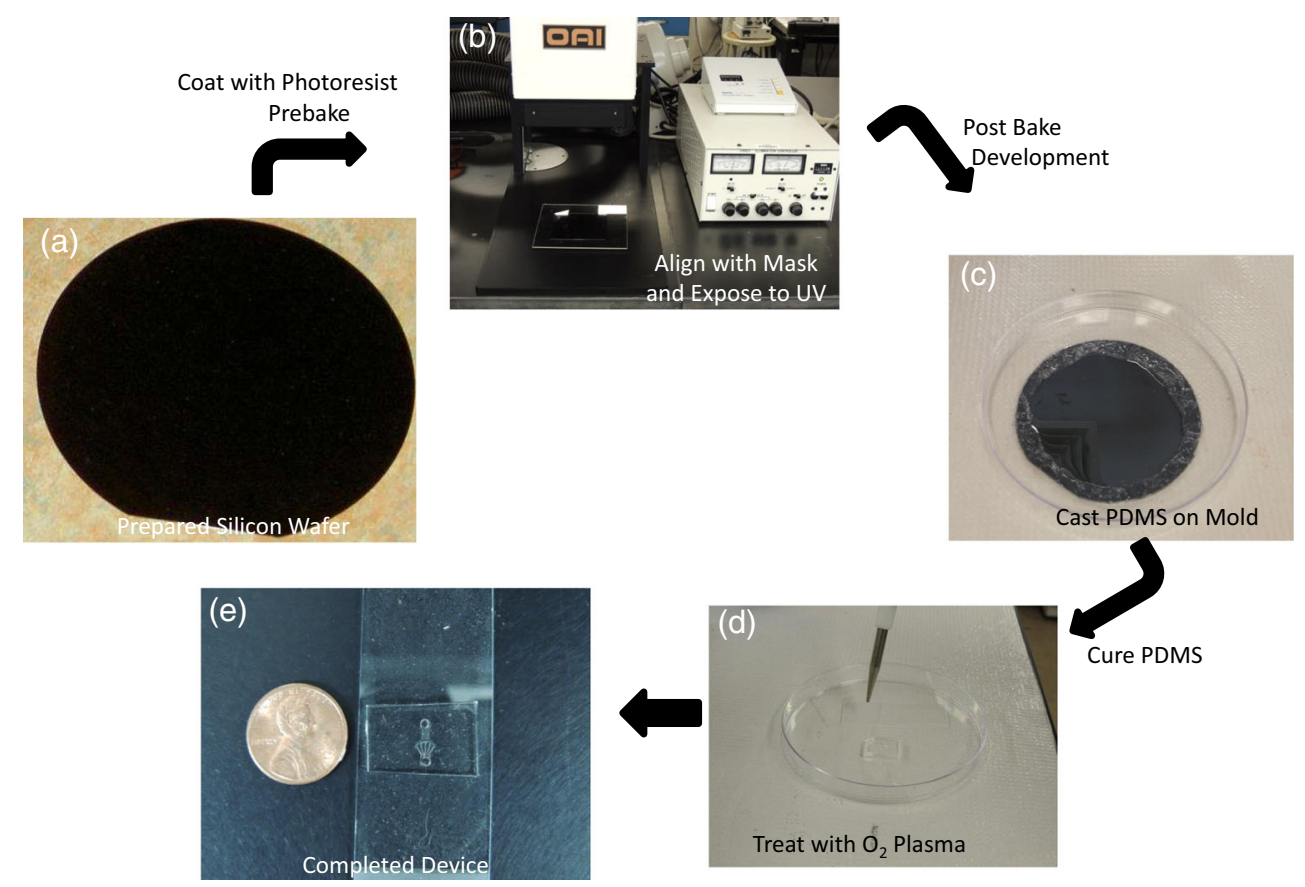

Fig. $1 \mu$ Fabrication processes used to construct the $\mu$ Retina system. a A prepared silicon wafer is spin coated with a thin, uniform film of SU-8 photoresist. b The coated wafer is then aligned and brought into close contact with a photomask consisting of quartz covered with a pattern of chrome. The UV light passes through the unchromed quartz to cross-link the photoresist in the underlying region, making the photoresist insoluble in the complementary developer solution. Development removes the

unexposed photoresist so that the desired microscale pattern remains on the wafer surface. c An elastomeric stamp utilizing a replica-molding technique is created by casting liquid PDMS on the relief of the pattern on the silicon wafer. As the PDMS cures, a precise negative pattern of the microscale pattern is created on its surface. $\mathbf{d}$ A microfluidics device is created by bonding the PDMS substrate containing the micropatterned features with a glass microscope slide. e A finished $\mu$ Retina

(Life Technologies) to model small molecule diffusion through the HA matrix. The concentration gradient within the arch compartment was then measured via fluorescence microscopy (Zeiss).

\subsection{Cell culture}

Multi-passage mouse retinal progenitor cells (RPCs) (Unachukwu et al. 2013) were cultured in polystyrene culture dishes in Neurobasal medium (NBM; Invitrogen-Gibco, Rockville, MD) containing $2 \mathrm{mM} \mathrm{L}$-glutamine, $100 \mathrm{mg} / \mathrm{ml}$ penicillin-streptomycin, $20 \mathrm{ng} / \mathrm{ml}$ epidermal growth factor (EGF; Invitrogen-Gibco) and neural supplement (B27 and N2; Invitrogen-Gibco), as per (Redenti et al. 2009). Cells were maintained in a bio-incubator at $37{ }^{\circ} \mathrm{C}$ and $5 \% \mathrm{CO}_{2}$, and media was refreshed every 3-4 days. 95\% Confluent cells were detached and utilized for immunofluorescence and device characterization.

\subsection{Immunofluorescence}

RPCs were seeded onto glass bottom chamber slides (Nunc Lab-tek II, Sigma-aldrich) coated with a poly-L-lysine and Laminin mix (.02 and $10 \mathrm{ug} / \mathrm{mL}$, respectively) at a cell density of $5 \times 10^{5}$ cells $/ \mathrm{mL}$. Cell samples were allowed to adhere for $6 \mathrm{~h}$, and then rinsed 3 times for $10 \mathrm{~min}$ each in PBS, blocked,

and permeabilized in PBS containing $10 \%$ goat serum, $1 \%$ BSA, and $0.1 \%$ Triton-X for $2 \mathrm{~h}$. Samples were then incubated with primary antibodies diluted in blocking buffer for $12 \mathrm{~h}$ at $4 \mathrm{C}$, the antibodies and their dilutions were as follows: Paired Box Gene 6 (Pax6) (Life Technologies) 1:20, Sine Oculis Homeobox Homolog 3 (Six3) (Fisher Scientific) 1:100, Orthodenticle Homeobox 2 (OTX2) (Fisher Scientific) 1:200, and cone-rod homeobox (CRX) (Life Technologies) 1: 100. Samples were then rinsed 3 times for 10 min each in PBS and Hoescht 33258 (Invitrogen) nuclear stain for $2 \mathrm{~h}$ at room temperature. The samples for Pax 6 and CRX were also incubated with a rhodamine-conjugated secondary antibody 1:800 (Life Technologies) during this time. Finally, samples were rinsed 3 times for 10 min each in PBS and sealed in mounting medium (Life Technologies) for epifluorescent imaging using a Leica confocal microscope.

\subsection{Boyden chamber Assay}

$700 \mu \mathrm{L}$ of cell culture media was added to each well of a 24 well plate. This was followed by the addition of $7 \mu \mathrm{L}$ of $100 \mathrm{ng} / \mathrm{mL}$ of SDF-1 two the test wells. A laminin-coated Bodyen insert (CytoSelect, Cell Biolabs) was added to each well. $300 \mu \mathrm{L}$ of cells at $10^{\wedge} 6$ cells $/ \mathrm{mL}$ were added to the upper chamber. The cells were then incubated overnight, for $17 \mathrm{~h}$. The cells were then fixed and stained for analysis. 


\subsection{Measurement of cell migration}

RPCs at a density of $5 \times 10^{5}$ cells $/ \mathrm{mL}$ were suspended within a $2 \%$ HA solution and injected into the arch compartment of the $\mu$ Retina. Cells were then incubated for $2 \mathrm{~h}$ at $37^{\circ} \mathrm{C}$ to allow for cell attachment to the matrix. After incubation, $20 \mu \mathrm{L}$ of SDF-1 was added to the source reservoir at either $100 \mathrm{ng} / \mathrm{mL}$ or $10 \mathrm{ng} / \mathrm{mL}$ concentration. The systems were then placed in an incubated, motorized stage of an inverted microscope (Nikon TE2000) with a 10× objective housed in a humidified incubator (Okolabs, NA, Italy) and imaged every $30 \mathrm{~min}$ for $18 \mathrm{~h}$. The temperature in the incubator was maintained at $37{ }^{\circ} \mathrm{C}$ with $5 \% \mathrm{CO}_{2} /$ balanced air supply. Live cell images of 50-60 cells/cell clusters throughout the arch compartment were obtained at 30-min intervals over an 18-h time periods for each SDF-1 test concentration.

\subsection{Data analysis}

Image analysis for the ICC images was performed utilizing NIS Elements (Nikon). Visual counting was used to determine total numbers of cells and numbers of cells expressing each marker in order to derive percent expression.

Parameters of cell motility $\left(\mathrm{M}_{\mathrm{o}}\right)$, maximum accumulated distance $\left(\mathrm{M}_{\mathrm{i}}\right)$ and average speed () for each cell tracked were resolved via The Manual Tracking and Chemotaxis and Migration Tool 2.0 (ibidi, Verona MI) plug-ins, all running on an ImageJ platform (NIH). Cell movement was tracked as a vector in polar coordinates of radius, $\mathrm{R}$, and angle, $\theta$. Maximum accumulated distance and average speed were compared between the two test concentrations of SDF-1.

A Student's $t$-test and least squares fit were used to measure and analyze the data using Matlab r2014a (The Mathworks, Inc). A least squares fit was calculated between the computational concentration profiles and experimentally measured profile. The $t$-test at $95 \%$ confidence was performed to determine the disparity between the two concentration gradients, where only p-values $<0.05$ were considered statistically significant.

\section{Results and discussion}

\subsection{Device design}

The $\mu$ Retina system was designed to generate a steady-state concentration profile within the arch compartment via diffusion. Two variations of the device were developed, one to mimic the mouse retina and the other to model the human retina, whose dimensions are shown in Table 1.

The human $\mu$ Retina system consists of a PDMS elastomer bonded to a glass microscope slide that houses a closed
Table 1 Clinical measurements of key components of the human and mouse visual system

\begin{tabular}{lll}
\hline Key features & Human eye & Mouse eye \\
\hline Length & $24 \mathrm{~mm}$ & $3.37 \mathrm{~mm}$ \\
Diameter & $28 \mathrm{~mm}$ & $3.32 \mathrm{~mm}$ \\
Aqueous humor & $260 \mu \mathrm{L}$ & $4.4 \mu \mathrm{L}$ \\
Vitreous humor & $5.2 \mathrm{~mL}$ & $5.3 \mu \mathrm{L}$ \\
Retinal arc & $32-51 \mathrm{~mm}$ & $3-5 \mathrm{~mm}$ \\
Retinal area & $1024 \pm 184 \mathrm{~mm}^{2}$ & $15.6 \mathrm{~mm}$ \\
Retinal subtense & $300 \mu \mathrm{m} / \mathrm{deg}$ & $31 \mu \mathrm{m} / \mathrm{deg}$ \\
\hline
\end{tabular}

microchannel and two fluidic reservoirs, a sink and a source, within the elastomer, as shown in Fig. 2b. The device is composed of three regions connected in series by 2 sets of microchannels. The top and bottom reservoirs are labeled as the source and sink, respectively, while the central compartment is comprised of an arch-shaped gradient $\mu$ channel. The source has a radius, $R_{i}$, of $4500 \mu \mathrm{m}$, and is connected to a set of 3 parallel inlet microchannels, each $200 \mu \mathrm{m}$ in thickness and $8000 \mu \mathrm{m}$ in length. These inlet channels feed into the top of the arch compartment. The arch compartment itself is a tapered construct, with a maximum width, $\mathrm{R}_{\mathrm{W}}$, of $400 \mu \mathrm{m}$ at the middle, and tapered symmetrically to a minimum width, $\mathrm{W}_{\text {end }}$, of $165 \mathrm{um}$ at both ends. The inner radius of the $\operatorname{arch}, \mathrm{R}_{\mathrm{C}}$, is $13500 \mu \mathrm{m}$ producing a total arc length of $42000 \mu \mathrm{m}$, which is within the range of measured values reported for the average human retina (Oyster 1999). The arch compartment both replicates the geometry of the human retina, as well as facilitates the creation of a controlled concentration gradient in the $\mathrm{R}$ and the $\theta$ directions. The arch compartment is connected to the sink reservoir by a set of 5 microchannels, $150 \mu \mathrm{m}$ wide and $8000 \mu \mathrm{m}$ long, that project radially from the sink reservoir and are spaced $15^{\circ}$ apart. The sink reservoir is a mirror image of the source reservoir with a radius, $\mathrm{R}_{\mathrm{O}}$, of $4500 \mu \mathrm{m}$ and a depth of $4 \mathrm{~mm}$. The volume of the entire system is $100 \mu \mathrm{L}$, confirmed via syringe pump. A large bridge channel is then manually cut $2 \mathrm{~mm}$ deep by $9 \mathrm{~mm}$ wide by $2 \mathrm{~cm}$ long along the top of the PDMS layer to fluidically connect the source and sink reservoirs.

The mouse $\mu$ Retina system, similarly to the human system, consists of a layer of PDMS bonded to a glass microscope slide with a closed microchannel contained within the PDMS layer, as shown in Fig. 2a. The device is composed of two compartments, a fluidic reservoir and an arch shaped $\mu$ channel. The reservoir is situated adjacent to the top of the arch. The arch has a maximum width, $\mathrm{R}_{\mathrm{W}}$, of $200 \mu \mathrm{m}$ in the middle and then tapers down symmetrically to a minimum width, $\mathrm{W}_{\text {end }}$, of $10 \mu \mathrm{m}$ at its two ends. The inner radius of the arch, $R_{C}$, is $1000 \mu \mathrm{m}$ providing a total arc length of $3140 \mu \mathrm{m}$, which is within the range of measured values reported for the average mouse retina (Remtulla and Hallett 1985). The total volume of the 


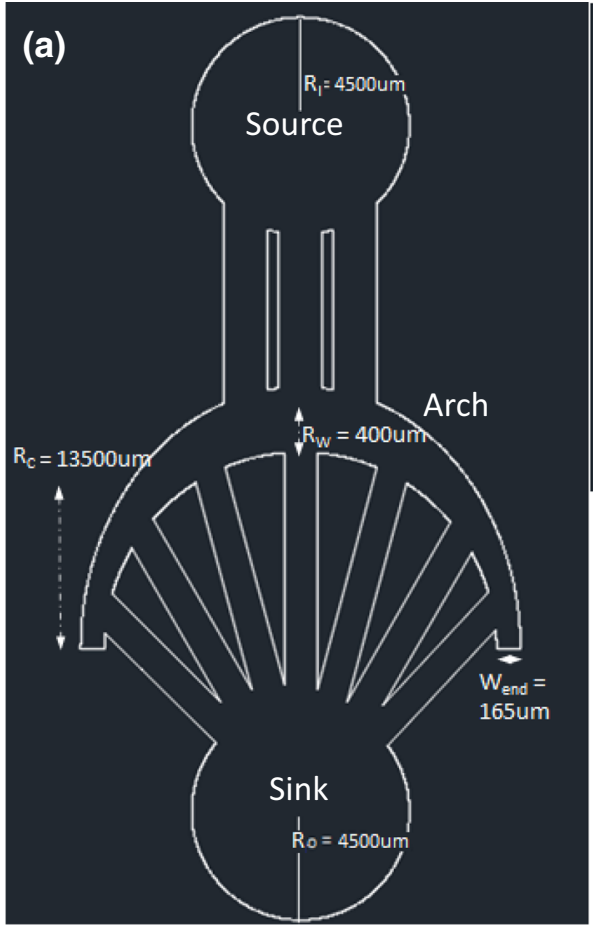

Fig. 2 Design of human and mouse $\mu$ Retina systems. a The human $\mu$ Retina device is composed of three compartments connected in series by a set of microchannels. The top and bottom compartments are labeled as the source and sink reservoir, respectively and are centered around an arch-shaped region. The source reservoir is located at the top of the device, with a radius, $\mathrm{R}_{\mathrm{i}}$, of $4500 \mu \mathrm{m}$, and is connected to a set of 3 parallel inlet microchannels, each $200 \mu \mathrm{m}$ in thickness and $8000 \mu \mathrm{m}$ in length. These inlet channels feed into the arch compartment. The arch is a tapered construct, with a maximum width, $\mathrm{R}_{\mathrm{W}}$, of $400 \mu \mathrm{m}$, and tapers symmetrically to a minimum width, $\mathrm{W}_{\text {end }}$, of $165 \mu \mathrm{m}$ at its ends. The

system is $10 \mu \mathrm{L}$. A geometric comparison of the two systems is provided in Table 2 .

\section{$3.2 \mu$ Retina operation}

The overall human $\mu$ Retina system works by using the large volume of the source and sink reservoirs to generate concentration gradients within the smaller volume of the arch compartment. Media containing cells in suspension is manually loaded into the bridge channel until the entire device is filled, followed by addition of the desired concentration of the chemical of interest into the source reservoir. As has been described in our labs previously developed microdevices (Kong et al. 2010), the large volume of the bridge channel and reservoirs compared to the arch compartment enables sustained transport within the channel for up to 7 days. To further extend this time scale and to further control the concentration gradient, a 3D HA matrix with density corresponding to that of the interphotorecepter matrix (IPM) present within the retina was added to the arch compartment. This serves not only to extend time-scales for experimentation, but also

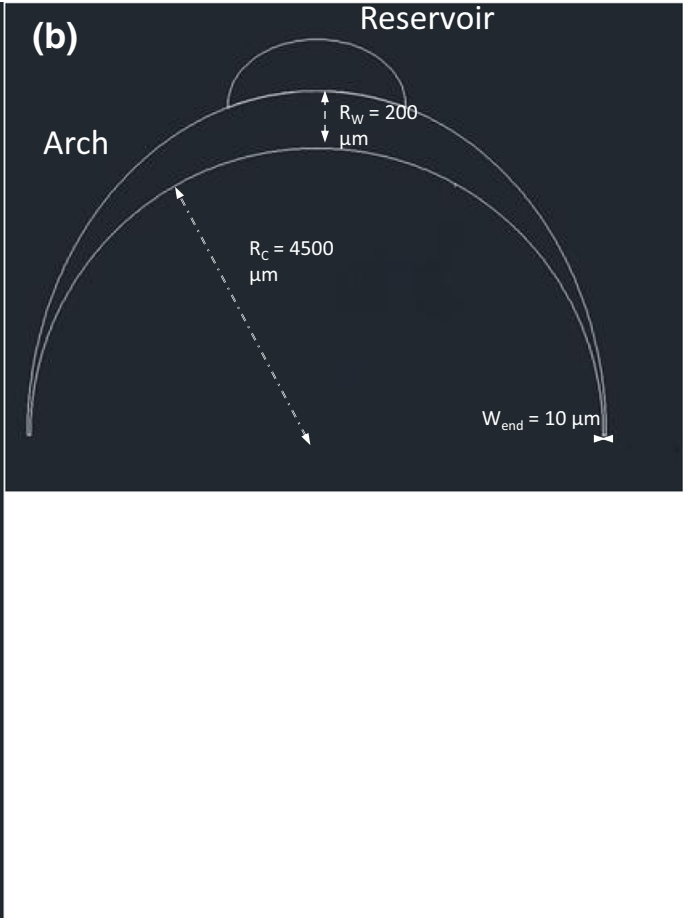

inner radius of the arch, $\mathrm{R}_{\mathrm{C}}$, is $13500 \mu \mathrm{m}$ providing a total arc length of $42000 \mu \mathrm{m}$. The arch compartment is connected to the sink reservoir, with radius $\mathrm{R}_{\mathrm{o}}=4500 \mu \mathrm{m}$, by 5 equally spaced channels, each $150 \mu \mathrm{m}$ thick and $8000 \mu \mathrm{m}$ in length. b The device is composed of a large reservoir compartment and an arch shaped gradient compartment. The reservoir is situated adjacent to the apex of the arch. The arch compartment has a maximum width, $\mathrm{R}_{\mathrm{W}}$, of $200 \mu \mathrm{m}$ and then tapers down symmetrically to a minimum width, $\mathrm{W}_{\text {end }}$, of $10 \mu \mathrm{m}$ at either ends. The inner radius of the arch, $\mathrm{R}_{\mathrm{C}}$, is $1000 \mu \mathrm{m}$

imposes a barrier to limit the effects of convective bulk flow due to hydrostatic differences (Mackenzie 1999). The use of HA results in a system, within the arch compartment, defined purely by Fick's laws of diffusion. As the system contains complex geometry, it was solved computationally utilizing Comsol Multiphysics 4.3. At each node point, the diffusion was characterized by the

Table $2 \mu$ Retina design: summary of key parameters within human and mouse $\mu$ Retina systems

\begin{tabular}{llll}
\hline Specifications & Symbol & $\begin{array}{l}\text { Human } \\
\mu \text { Retina }(\mu \mathrm{m})\end{array}$ & $\begin{array}{l}\text { Mouse } \\
\mu \text { Retina }(\mu \mathrm{m})\end{array}$ \\
\hline Inner radius & $\mathrm{R}_{\mathrm{C}}$ & 13500 & 1000 \\
Min width & $\mathrm{W}_{\mathrm{end}}$ & 165 & 10 \\
Max width & $\mathrm{R}_{\mathrm{W}}$ & 400 & 200 \\
Height & $\mathrm{H}$ & 110 & 110 \\
Total volume & $\mathrm{V}_{\mathrm{T}}$ & $125 \mu \mathrm{L}$ & $10 \mu \mathrm{L}$ \\
Inlet & $\mathrm{R}_{\mathrm{i}}$ & 4500 & $\mathrm{~N} / \mathrm{A}$ \\
Outlet & $\mathrm{R}_{\mathrm{o}}$ & 4500 & $\mathrm{~N} / \mathrm{A}$ \\
Steady state timing & $\mathrm{T}_{\mathrm{s}}$ & $1800 \mathrm{~s}$ & $360 \mathrm{~s}$ \\
\hline
\end{tabular}


mass balance equation, shown in Eq. 1:

$\frac{\delta n(x, t)}{\delta t}+u \cdot \nabla c=\nabla \cdot(D \nabla n(x, t))$

where $n(x, t)$ is the concentration of the diffusing material at the location $\mathrm{x}=(\mathrm{x}, \mathrm{y})$ and time, t. $\mathrm{D}$ denotes the diffusion coefficient for the diffusing species, and $u$ is the velocity vector at the location $\mathrm{x}$ and time $\mathrm{t}$. The first term on the left-hand side corresponds to the accumulation of the species. The second term is the convective term due to velocity field $u$. The right-hand side defines the diffusion transport. Measurements of the flow of microbeads within both $\mu$ Retina systems demonstrated negligible bulk flow, eliminating the $u$ term to reduce the mass balance to Eq. 2, shown below:

$\frac{\delta n(x, t)}{\delta t}=\nabla \cdot(D \nabla n(x, t))$

The elegant design of our system enables a straightforward and unforced setup. That is, after loading there is no need for further instruments to establish a chemical concentration gradient. Furthermore, the unique geometry facilitates symmetric diffusion within the arch compartment.
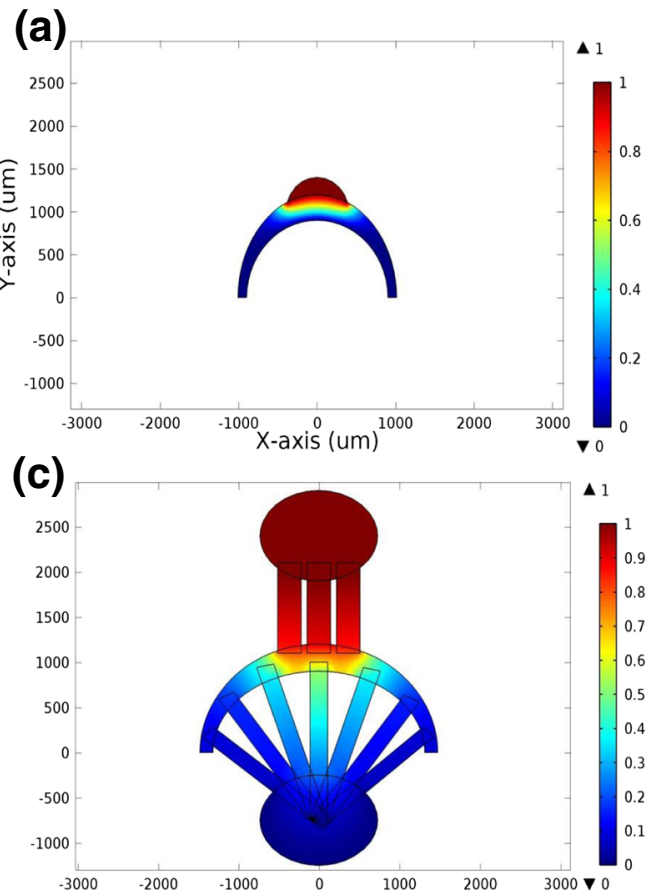

Fig. 3 Computer derived concentration profile within mouse and human $\mu$ Retina devices. a Steady-state concentration profile for SDF-1 within the mouse $\mu$ Retina device $\mathbf{b}$ The concentration profile within the mouse $\mu$ Retina, traced at the arch midline along the $\theta$ dimension. The concentration profile exhibits an exponential decay approaching the terminal ends of the arch. This decay is symmetric about the center line of the arch compartment. $\mathbf{c}$ Steady-state concentration profile for SDF-1

\subsection{Validation of microenvironments}

The concentration profiles developed in the $\mu$ Retina system were modeled computationally and verified experimentally, as seen in Fig. 3. The computational model indicates that the concentration profile, traced at a constant radius along theta, $\theta$, exhibits an exponential decay approaching the terminal ends of the device, Fig. $3 \mathrm{c}-$ d. This decay is symmetric about the center line of the arch compartment. This indicates a change in concentration of several orders of magnitude in the concentration gradient along the compartment. Furthermore, the computational model indicates that the concentration profile reaches steady-state in $1800 \mathrm{~s}$ in the human system and $360 \mathrm{~s}$ in the mouse, as shown in Table 2 .

Computational results were validated against experimental measurements within the fabricated $\mu$ Retina using a model fluorophore (Dextran) to mimic SDF-1. The fluorescent intensity of dextran was measured, as a representative of the concentration, from the apex of the $\mu$ Retina arch to the terminal ends on either side after establishing steady-state. Figure $3 \mathrm{~d}$ shows the computational projection, dashed, plotted alongside experimental observations, solid. As shown, the experimental data has a correlation
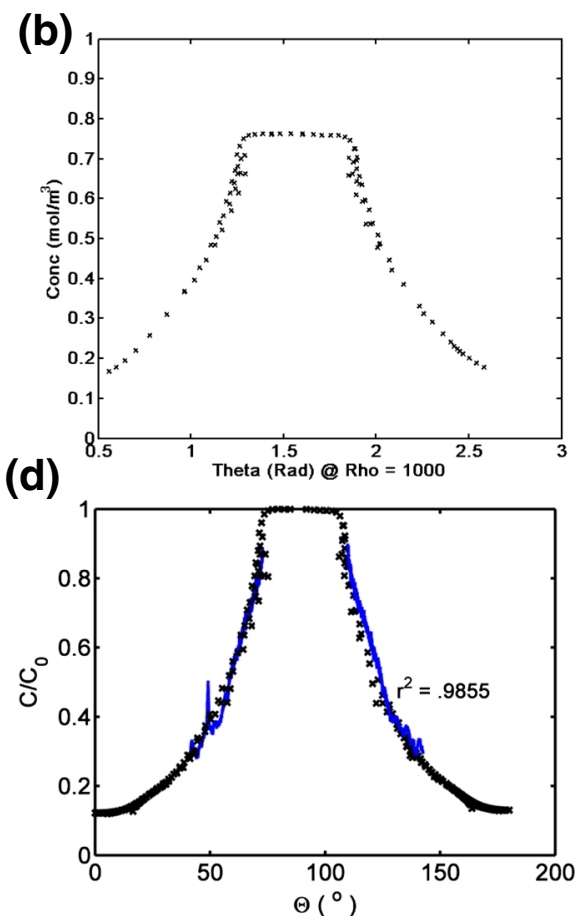

within the human $\mu$ Retina device $\mathbf{d}$ Computational concentration profile within the human $\mu$ Retina, dotted line, traced at a constant radius along the $\theta$, exhibits an exponential decay approaching the terminal ends of the device. This decay is symmetric about the center line of the arch compartment. The solid line shows the experimental measurements for the concentration gradient profile within the $\mu$ Retina using a tagged Dextran model 
Fig. 4 RPCs in vitro culture within the $\mu$ Retina device after $48 \mathrm{~h}$. a and b RPCs on HA within the $\mu$ Retina device. The line at the bottom of each image is the edge of the arch compartment. Scale bar $=50 \mu \mathrm{m} \mathbf{c}$ RPCs on HA in a polystyrene culture dish

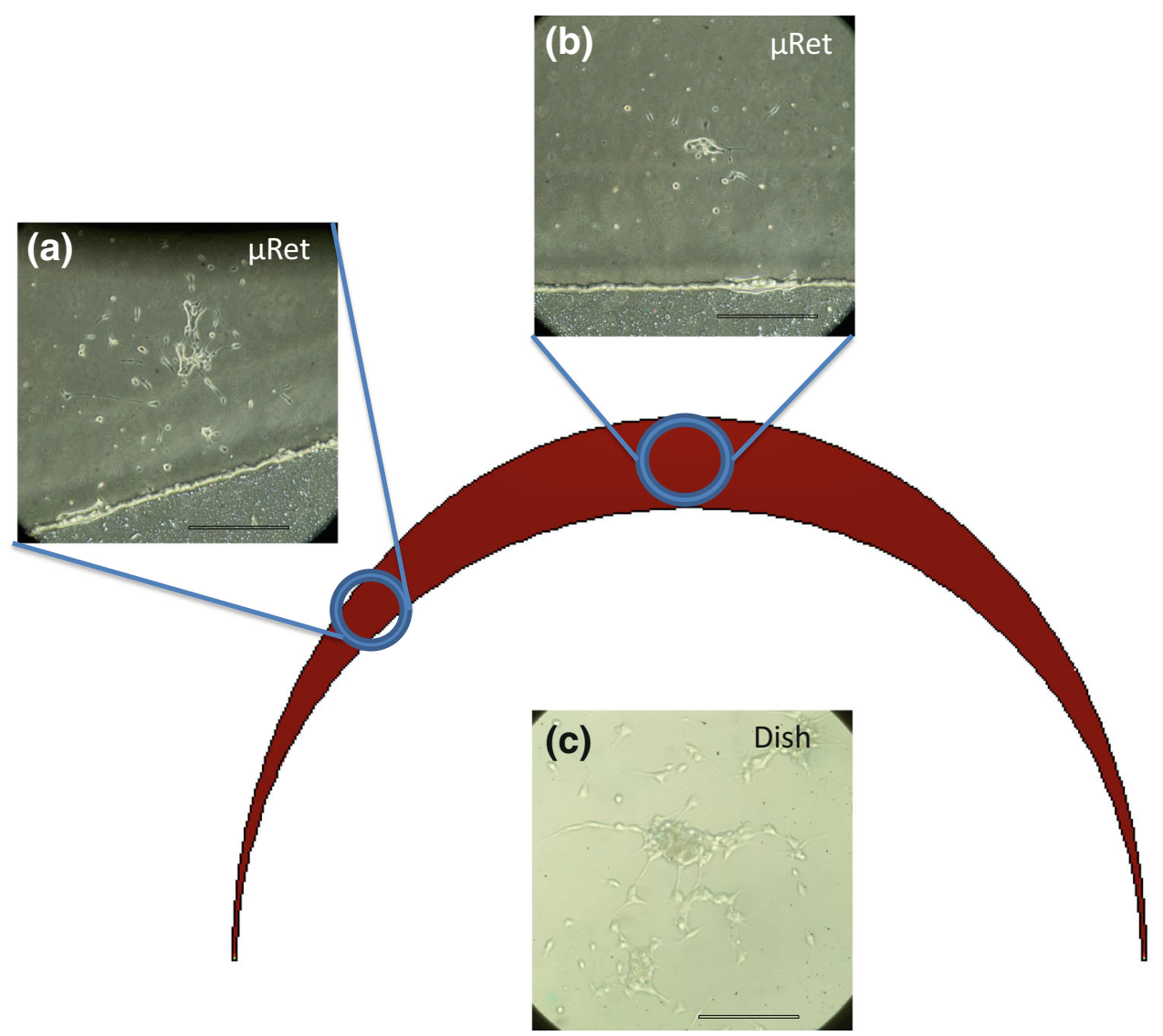

coefficient of $\mathrm{R}^{2}=0.9855$ with the predictions of the computational model (Fig. 3d).

\subsection{Cell viability, morphology and lineage}

An initial set of experiments was performed to examine cell viability, morphology, and clustering within our $\mu$ Retina system for prolonged periods of time. Here, RPCs were injected within a 3D HA matrix into the device and monitored for $48 \mathrm{~h}$. Images in Fig. 4 illustrate cells within different sections of the device after $48 \mathrm{~h}$, as well as images of cells cultured in HA within tissue culture flasks. The cells appear in small clusters with dendritic like cells projecting away from small clusters in both cases. Cell shape index (CSI) analysis, shown in Table 3, illustrates that cells are elongated with an average CSI of .217. This is comparable to what is seen on $\mathrm{HA}$ in the culture dishes, average $\mathrm{CSI}=.353$, suggesting that there is minimal confinement effect on cell morphology.

ICC experiments were next employed to examine four specific markers of cell lineage for the retinal RPCs. Fig. 5 illustrates that 96.67 and $91.56 \%$ of cell samples expressed the markers Pax 6 and Six3, respectively, to indicate that RPCs were multipotent. Further, $97.72 \%$ of samples additionally expressed the OTX2 marker, which suggests that the RPCs may be developing toward photoreceptor cell fates. Lastly, $15.79 \%$ of OTX $(+)$ cell samples expressed CRX, which suggests development toward photoreceptor fates. The limited expression of this maker along with the strong presence of OTX2, Pax6 and Six3 verify that the RPC samples have the potential to acquire photoreceptor precursor fate. A number of studies have transplanted RPC with comparable ICC profiles (Steward 1958).

Table 3 Cell shape index

\begin{tabular}{ll}
\hline Coating & Average CSI \\
\hline HA: Dish & $0.353 \pm 0.108$ \\
Laminin: dish & $0.567 \pm 0.088$ \\
Polystyrenel: dish & $0.481 \pm 0.023$ \\
HA:uRetina & $0.217 \pm 0.043$
\end{tabular}

Average values of CSI of RPCs within the human $\mu$ Retina and culture flasks. Three substrates were used in the culture flasks. HA and Laminin, two ECM components regularly found in the IPM and polystyrene as a control. HA was used in the $\mu$ Retina device. 


\subsection{Measurement of cell migration}

The final set of experiments used the $\mu$ Retina system to examine the migration of RPC cells within controlled microenvironments of SDF-1. SDF-1 was selected because Boyden chamber migration assays demonstrated the effectiveness of SDF-1 as an effective chemotaxic agent for RPCs, as seen in Fig. 6. SDF-1 was tested for
Fig. 5 Markers of cell lineage for retinal progenitor cells. a Cell stained for Pax 6 expression (red) and b Six 3 (green), respectively, indicate that cells were of the retinal progenitor cell lineage. c Samples additionally expressing OTX2 (green), which indicates that the cells were in the Early Photoreceptor Progenitor stage. d Samples stained for CRX expression, which denotes terminally-differentiated rod and cone photoreceptor cells. e Control samples stained with the $2^{\circ}$ antibody, to check for nonspecific binding. All nuclei were stained with DAPI (blue)

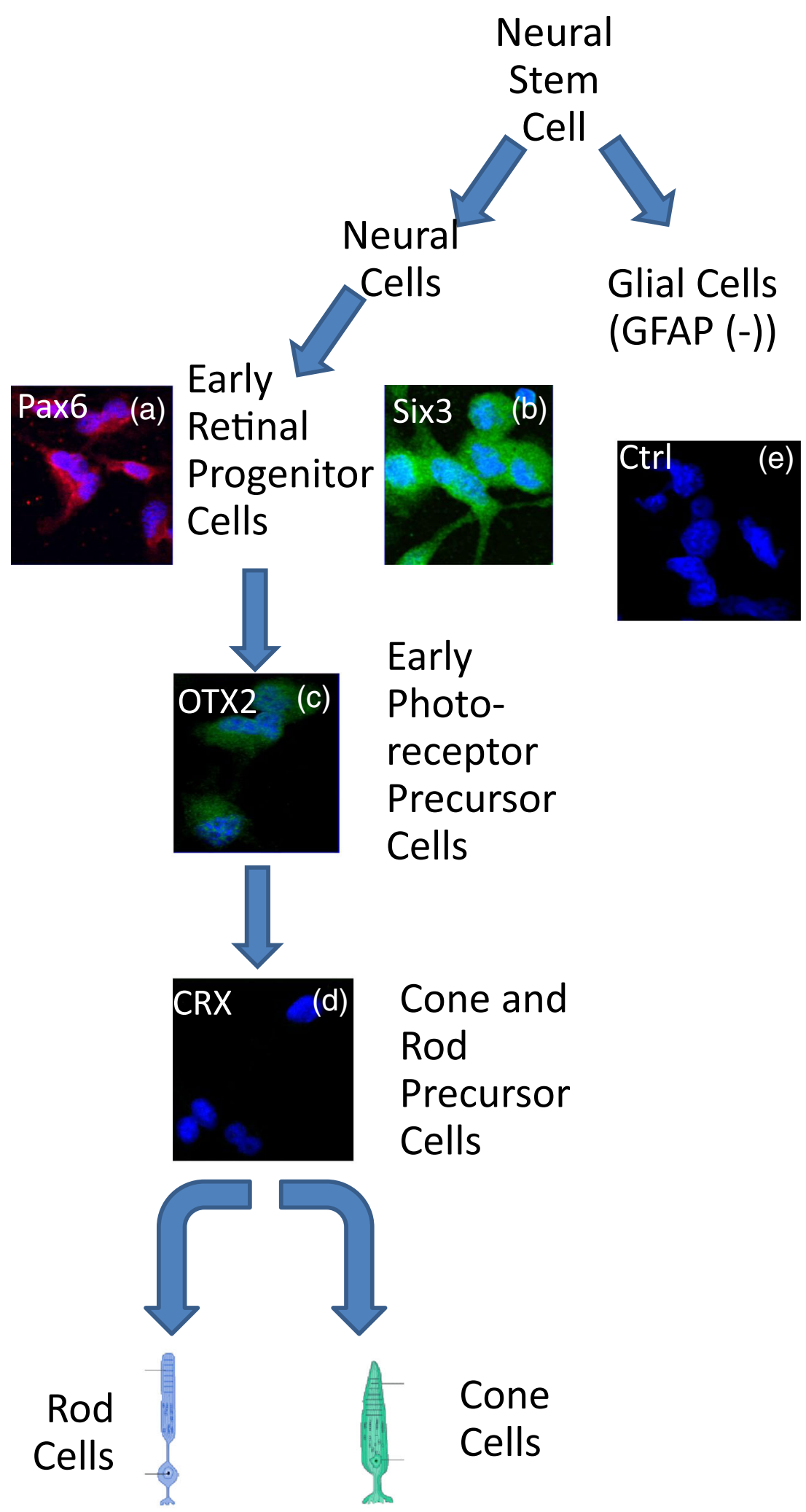




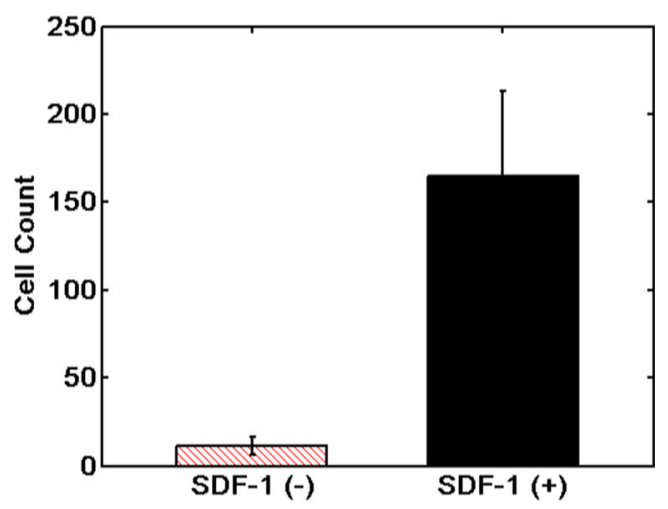

Fig. 6 Boyden chamber analysis for RPC under the Influence of SDF-1. Adding SDF-1 to the Boyden chamber resulted in a significant upswing in the number of migrating cells, compared to the control, indicating it is a potent chemotactic agent for RPCs

motility induction at a concentration of $100 \mathrm{ng} / \mathrm{mL}$. Using Student's $t$-test, significant increases in RPC migration were observed with the administration of $100 \mathrm{ng} / \mathrm{mL}$ SDF-1, 165 \pm 47.958 cells/well, over the control, $11.4 \pm$ 5.12 cells/well $(n=5$ Boyden chambers, with a p-value of .002), as seen in Fig. 6. Cells were inserted into the $\mu$ Retina and allowed to adhere before SDF- 1 was injected into the source reservoir of the system, $R_{s}$, and transported within the $\mu$ Retina to generate the concentration profiles validated in Fig. 3c. As shown in Fig. 7, no significant RPC cell movement was seen over an 18 -h period within concentration profiles generated by $10 \mathrm{ng} /$ $\mathrm{mL}$ of SDF-1 in the system. By contrast, RPCs were seen

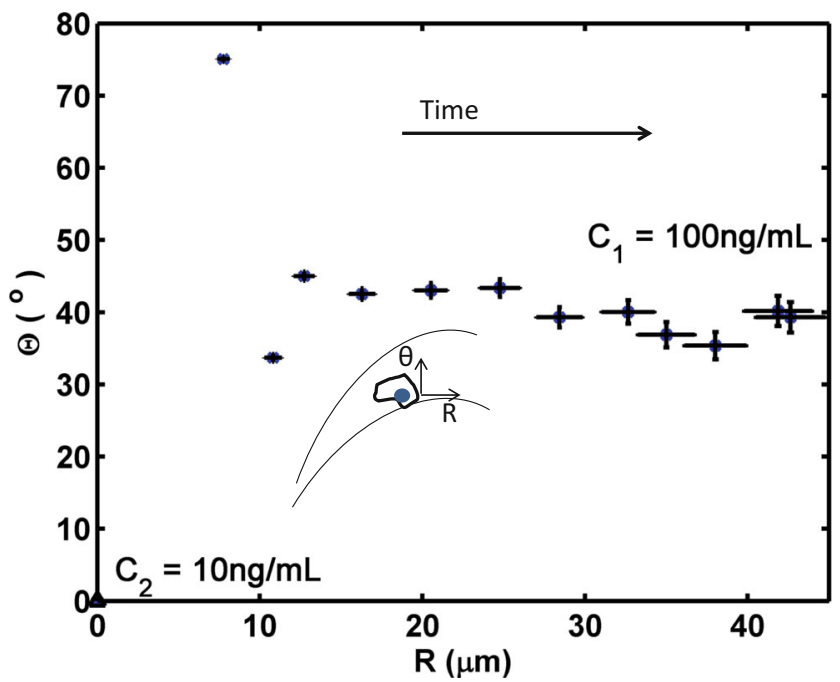

Fig. 7 Cell Migration in Response to SDF-1 Concentration Gradients. Circles indicate the average position of cells subject to concentration gradients generated by addition of $100 \mathrm{ng} / \mathrm{mL}$ SDF-1. Triangles indicate the average position of cells exposed to concentration gradients generated by addition of $10 \mathrm{ng} / \mathrm{mL}$ SDF- 1 . Cells subjected to $10 \mathrm{ng} / \mathrm{mL}$ exhibited no displacement throughout the $18 \mathrm{~h}$ of the migration study. Conversely, the cells subjected to $100 \mathrm{ng} / \mathrm{mL}$ showed correlated movement towards the regions of higher concentration of SDF-1. These cells displayed an average movement of $2.83 \pm 0.96 \mu \mathrm{m} / \mathrm{s}$ to migrate in a very directional manner in $(R, \theta)$ towards increasing concentration gradients when $100 \mathrm{ng} / \mathrm{mL}$ of SDF-1 were used. As shown, cell trajectories were along the theta direction, remaining linear with $\mathrm{R}$. The values of average cell speed, $\mathrm{S}$, and maximum accumulated distance per cell at $100 \mathrm{ng} / \mathrm{mL}$ illustrate that there is significant directed movement, averaging $34.6 \mathrm{um} \pm 1.7 \mathrm{um}$ over $18 \mathrm{~h}$ with an average velocity of $2.83 \mathrm{um} / \mathrm{h} \pm .096 \mathrm{um} / \mathrm{h}$. Initially the cells move primarily through $\theta$, followed by movement primarily in the $\mathrm{R}$ direction. Figure 3 corroborates this, as at the edges of the arch compartment, the concentration gradient is changing largely with $\theta$ dependence, and at the plateau region the concentration gradient no longer depends on $\theta$ but solely upon R. At $10 \mathrm{ng} / \mathrm{ml}$ there was zero measurable movement of RPCs throughout the $18 \mathrm{~h}$ study. Since the matrix replicates the density and diffusion characteristics of the retina, this data provides insight into the anticipated behavior of RPCs within a SDF-1 primed retina. The stark difference in the behavior between the two concentration gradients indicates that there is a threshold concentration at which RPCs respond, suggesting a potential mechanism by which to manipulate RPC migration. As the system is based purely on diffusion, it enables numerous combinations of cytokines and small molecules to be used to manipulate and tune RPC migration through desired concentration gradients. However, to determine the extent of the biphasic behavior or concentration dependence, the migration at additional concentrations must be measured.

\section{Conclusions}

We have developed a novel and convenient biomimetic microfluidics device to exam the cell migration behavior within the geometry of the human and mouse retina. The design details a novel platform in the ophthalmologic field, providing a model system through which to study behavior of PR cells. Coupled computer simulations and experimental validations characterized and confirmed the formation of chemical concentration gradients within the $\mu$ Retina, while $\mathrm{R}-\theta$ imagers captured cell migration in response to high and low concentration gradients of SDF-1. The $\mu$ Retina system demonstrated dual behavior between the two concentrations: The low concentration illustrated no movement while the high concentration showed significant directed movement along increasing concentration gradient, indicative of chemotaxis. Divergent cell behavior between the concentration gradients illustrates that, to some degree, retinal cell migration within the retina can be tuned by utilizing cytokine gradients. This initial data is an encouraging step toward utilizing microtechnology to improve our understanding and treatment of retinal diseases. 


\section{References}

E. Margalit, S.R. Sadda, Retinal and optic nerve diseases. Artif. Organs 27, 963-974 (2003)

C. Rivolta, D. Sharon, M.M. DeAngelis, T.P. Dryja, Retinitis pigmentosa and allied diseases: numerous diseases, genes, and inheritance patterns. Hum. Mol. Genet. 11, 1219-1227 (2002)

A.D. Kulkarni, B.D. Kuppermann, Wet age-related macular degeneration. Adv. Drug Deliv. Rev. 57, 1994-2009 (2005)

H.J. Klassen, T.F. Ng, Y. Kurimoto, I. Kirov, M. Shatos, P. Coffey, M.J. Young, Multipotent retinal progenitors express developmental markers, differentiate into retinal neurons, and preserve light-mediated behavior. Invest. Ophthalmol. Vis. Sci. 45, 4167-4173 (2004)

R.E. MacLaren, R. Pearson, A. MacNeil, R. Douglas, T. Salt, M. Akimoto, A. Swaroop, J. Sowden, R. Ali, Retinal repair by transplantation of photoreceptor precursors. Nature 444, 203-207 (2006)

S. Redenti, W.L. Neeley, S. Rompani, S. Saigal, J. Yang, H. Klassen, R. Langer, M.J. Young, Engineering retinal progenitor cell and scrollable poly (glycerolsebacate) composites for expansion and subretinal transplantation. Biomaterials 30, 3405-3414 (2009)

J.S. Meyer, M.L. Katz, J.A. Maruniak, M.D. Kirk, Embryonic stem cell derived neural progenitors incorporate into degenerating retina and enhance survival of host photoreceptors. Stem Cells 24, 274-283 (2006)

S. Tao, C. Young, S. Redenti, Y. Zhang, H. Klassen, T. Desai, M.J. Young, Survival, migration and differentiation of retinal progenitor cells transplanted on micromachined poly (methyl methacrylate) scaffolds to the subretinal space. Lab Chip 7, 695-701 (2007)

M. Tomita, E. Lavik, H. Klassen, T. Zahir, R. Langer, M.J. Young, Biodegradable polymer composite grafts promote the survival and differentiation of retinal progenitor cells. Stem Cells 23, 1579-1588 (2005)

T.F. Ng, E. Lavik, H. Keino, A.W. Taylor, R.S. Langer, M.J. Young, Creating an immune privileged site using retinal progenitor cells and biodegradable polymers. Stem Cells 25, 1552-1559 (2007)

J. Lakowski, M. Baron, J. Bainbridge, A. Barber, R. Pearson, R. Ali, J. Sowden, Cone and rod photoreceptor transplantation in models of the childhood retinopathy Leber congenital amaurosis using flowsorted Crx-positive donor cells. Hum. Mol. Genet. ddq378 (2010)

E. West, R. Pearson, R. MacLaren, J. Sowden, R. Ali, Cell transplantation strategies for retinal repair. Prog. Brain Res. 175, 3-21 (2009)

P.E. Nickerson, K.M. Ronellenfitch, N.F. Csuzdi, J.D. Boyd, P.L. Howard, K.R. Delaney, R.L. Chow, Live imaging and analysis of postnatal mouse retinal development. BMC Dev. Biol. 13, 24 (2013)

C. Mohlin, K. Johansson, Death of photoreceptors in organotypic retinal explant cultures: implication of rhodopsin accumulation and endoplasmic reticulum stress. J. Neurosci. Methods 197, 56-64 (2011)

I. Meyvantsson, D.J. Beebe, Cell culture models in microfluidic systems. Annu. Rev. Anal. Chem. 1, 423-449 (2008)

F. Morin, N. Nishimura, L. Griscom, B. LePioufle, H. Fujita, Y. Takamura, E. Tamiya, Constraining the connectivity of neuronal networks cultured on microelectrode arrays with microfluidic techniques: a step towards neuron-based functional chips. Biosens. Bioelectron. 21, 1093-1100 (2006)

J. Park, H. Koito, J. Li, A. Han, A multi-compartment CNS neuron-glia coculture microfluidic platform. J. Vis. Exp.: JoVE (2009)

T. Stieglitz, M. Schuettler, J.-U. Meyer, Micromachined, polyimide-based devices for flexible neural interfaces. Biomed. Microdevices 2, 283 $294(2000)$

S. Hosmane, I.H. Yang, A. Ruffin, N. Thakor, A. Venkatesan, Circular compartmentalized microfluidic platform: study of axon-glia interactions. Lab Chip 10, 741-747 (2010)

H.J. Kim, J.W. Park, J.W. Park, J.H. Byun, B. Vahidi, S.W. Rhee, N.L. Jeon, Integrated microfluidics platforms for investigating injury and regeneration of CNS axons. Ann. Biomed. Eng. 40, 1268-1276 (2012)

J.W. Park, B. Vahidi, A.M. Taylor, S.W. Rhee, N.L. Jeon, Microfluidic culture platform for neuroscience research. Nat. Protoc. 1, 2128$2136(2006)$

B. Vahidi, J.W. Park, H.J. Kim, N.L. Jeon, Microfluidic-based strip assay for testing the effects of various surface-bound inhibitors in spinal cord injury. J. Neurosci. Methods 170, 188-196 (2008)

P.S. Dittrich, A. Manz, Lab-on-a-chip: microfluidics in drug discovery. Nat. Rev. Drug Discov. 5, 210-218 (2006)

R.S. Shawgo, A.C. Richards Grayson, Y. Li, M.J. Cima, BioMEMS for drug delivery. Curr. Opinion Solid State Mater. Sci. 6, 329-334 (2002)

D.A. Lavan, T. McGuire, R. Langer, Small-scale systems for in vivo drug delivery. Nat. Biotechnol. 21, 1184-1191 (2003)

S. Estermann, K. Yuttitham, J.A. Chen, O.-T. Lee, R.L. Stamper, Comparative in vitro flow study of 3 different Ex-PRESS miniature glaucoma device models. J. Glaucoma 22, 209-214 (2013)

T. Pan, M.S. Stay, V.H. Barocas, J.D. Brown, B. Ziaie, Modeling and characterization of a valved glaucoma drainage device with implications for enhanced therapeutic efficacy. IEEE Trans. Biomed. Eng. 52, 948-951 (2005)

B. Schlosshauer, A. Hoff, E. Guenther, E. Zrenner, H. Ha, Towards a retina prosthesis model: neurons on microphotodiode arrays in vitro. Biomed. Microdevices 2, 61-72 (1999)

P. Camelliti, J.O. Gallagher, P. Kohl, A.D. McCulloch, Micropatterned cell cultures on elastic membranes as an in vitro model of myocardium. Nat. Protoc. 1, 1379-1391 (2006)

C.M. Puleo, W.M. Ambrose, T. Takezawa, J. Elisseeff, T.-H. Wang, Integration and application of vitrified collagen in multilayered microfluidic devices for corneal microtissue culture. Lab Chip 9, 3221-3227 (2009)

J. Park, K.B. Kim, J. Lee, H.C. Kim, D. Huh, Organomimetic microsystems technologies. Biomed. Eng. Lett. 2, 88-94 (2012)

M.R. Steedman, S.L. Tao, H. Klassen, T.A. Desai, Enhanced differentiation of retinal progenitor cells using microfabricated topographical cues. Biomed. Microdevices 12, 363-369 (2010)

Q. Kong, R.J. Majeska, M. Vazquez, Migration of connective tissue-derived cells is mediated by ultra-low concentration gradient fields of EGF. Exp. Cell Res. 317, 1491-1502 (2011)

U.J. Unachukwu, M. Sauane, M. Vazquez, S. Redenti, Microfluidic generated EGF-gradients induce chemokinesis of transplantable retinal progenitor cells via the JAK/STAT and PI3kinase signaling pathways. PLoS ONE 8, e83906 (2013)

Q. Kong, R.A. Able, V. Dudu, M. Vazquez, A microfluidic device to establish concentration gradients using reagent density differences. J. Biomech. Eng. 132, 121012 (2010)

A. Hussain, C. Starita, A. Hodgetts, J. Marshall, Macromolecular diffusion characteristics of ageing human Bruch's membrane: implications for age-related macular degeneration (AMD). Exp. Eye Res. 90, 703-710 (2010)

L. Pitkänen, V.-P. Ranta, H. Moilanen, A. Urtti, Permeability of retinal pigment epithelium: effects of permeant molecular weight and lipophilicity. Invest. Ophthalmol. Vis. Sci. 46, 641-646 (2005)

C.W. Oyster, The Human Eye (Sinauer, Sunderland, 1999)

S. Remtulla, P. Hallett, A schematic eye for the mouse, and comparisons with the rat. Vis. Res. 25, 21-31 (1985)

J. Mackenzie, Uniform convergence analysis of an upwind finite-difference approximation of a convection-diffusion boundary value problem on an adaptive grid. IMA J. Numer. Anal. 19, 233-249 (1999)

F. Steward, Growth and organized development of cultured cells. III. Interpretations of the growth from free cell to carrot plant. Am. J. Bot. 709-713 (1958) 